\title{
Estratégias utilizadas pelo enfermeiro da atenção primária na educação em saúde de hipertensos: revisão integrativa
}

\author{
Strategies used by primary care nurses in hypertensive health education: integrative review \\ Estrategias utilizadas por enfermeras de atención primaria en educación sanitaria para \\ pacientes hipertensos: una revisión integradora
}

Felipe Antonio Cunha dos Santos ${ }^{1 *}$, Andyara do Carmo Pinto Coelho Paiva ${ }^{1}$, Adélia Dayane Guimarães Fonseca ${ }^{1}$, Flávia Batista Barbosa de Sá Diaz², Letícia de Cássia Assis Gonçalves ${ }^{1}$, Letícia Rocha Marques Zanela1, Thaís Vasconselos Amorim¹, Luciano Côrtes Paiva ${ }^{3}$, Juliana Bernardo Nazareth ${ }^{1}$, Anna Maria de Oliveira Salimena ${ }^{1}$.

\section{RESUMO}

Objetivo: Conhecer as estratégias empregadas pelo enfermeiro da atenção primária na educação em saúde de hipertensos. Métodos: Trata-se de uma revisão integrativa, com buscas realizadas entre os meses de setembro e outubro de 2020, nas bases de dados Cumulative Index to Nursing and Allied Health Literature, Medical Literature Analysis and Retrieval System Online, Literatura Latino-Americana e do Caribe em Ciências de Saúde e Scientific Electronic Library Online com os descritores: educação em saúde, enfermagem, hipertensão e atenção primária à saúde. Resultados: As estratégias utilizadas pelo enfermeiro para realizar atividades educativas em saúde direcionadas aos hipertensos são: estratégias grupais, sala de espera, visita domiciliar e a consulta do Hiperdia. Os estudos revelam que, muitas vezes, essa educação ainda é pautada na transmissão vertical e ressaltam sobre a importância do estabelecimento do vínculo e do diálogo para que a sensibilização da pessoa com HAS seja efetiva. Considerações finais: Os modelos tradicionais, centrados no modelo biomédico, ainda são preponderantes nas práticas educativas. Os enfermeiros, inseridos nos cenários da atenção primária, precisam conhecer e se empoderar de outras estratégias educativas.

Palavras-chave: Educação em saúde, Enfermagem, Hipertensão, Atenção primária à saúde.

\begin{abstract}
Objective: Know the strategies used by primary care nurses in health education for hypertensive patients. Methods: This is an integrative review, with searches carried out between September and October 2020, in the Cumulative Index to Nursing and Allied Health Literature, Medical Literature Analysis and Retrieval System Online, Literatura Latino-Americana e do Caribe em Ciências de Saúde e Scientific Electronic Library Online databases, with the following descriptors: health education, nursing, Hypertension and Primary Health Care. Results: The strategies used by nurses to carry out health educational activities aimed at hypertensive patients are: group strategies, waiting room, home visit and Hiperdia consultation. Studies reveal that, often, this education is still based on vertical transmission and emphasizes the importance of establishing a bond and dialogue so that the awareness of the person with SAH is effective. Final considerations: The traditional models, centered on the biomedical model, with a focus on banking education, are still predominant in educational practices. Nurses, inserted in Primary Health Care scenarios, need to know and empower themselves with other educational strategies.
\end{abstract}

Keywords: Health education, Nursing, Hipertension, Primary health care.

\section{RESUMEN}

Objetivo: Conocer las estrategias que utilizan los enfermeros de atención primaria en la educación para la salud del paciente hipertenso. Métodos: Se trata de una revisión integradora, con búsquedas realizadas entre los meses de septiembre y octubre de 2020, en las bases de datos Cumulative Index to Nursing and Allied Health Literature, Medical Literature Analysis and Retrieval System Online, Latin American Literature and the Caribbean in Health Sciences y Scientific Electronic Library Online con los descriptores: educación en salud, enfermería, hipertensión y atención primaria de salud. Resultados: Las estrategias que utilizan los enfermeros para realizar actividades de educación en salud dirigidas a pacientes hipertensos son: estrategias grupales, sala de espera, visitas domiciliarias y consulta de Hiperdia. Los estudios revelan que, a menudo, esta educación todavía se basa en la transmisión vertical y enfatizan la importancia de establecer un vínculo y un diálogo para que la sensibilización de las personas con HAS sea efectiva. Consideraciones finales: Los modelos tradicionales, centrados en el modelo biomédico, aún predominan en las prácticas educativas. El enfermero, inserto en el ámbito de la atención primaria, necesita conocer y empoderarse con otras estrategias educativas.

Palabras clave: Educación para la salud, Enfermería, Hipertensión, Atención primaria de salud.

\footnotetext{
${ }^{1}$ Universidade Federal de Juiz de Fora (UFJF), Juiz de Fora - MG. *E-mail: Iuandyjf@yahoo.com.br

2 Universidade Federal de Viçosa (UFV), Viçosa - MG.

${ }^{3}$ Hospital Santa Casa de Misericórdia, Juiz de Fora - MG.
} 


\section{INTRODUÇÃO}

A Hipertensão Arterial Sistêmica (HAS) é uma patologia clínica que tem como característica o aumento dos níveis pressóricos $\geq 140$ e/ou $90 \mathrm{mmHg}$. Geralmente está ligada a outros distúrbios metabólicos e se agrava quando somada a fatores como obesidade, dislipidemias e diabetes. Esse quadro compromete o aparelho cardiovascular e respiratório podendo ocasionar o Infarto Agudo do Miocárdio (IAM), Acidente Vascular Encefálico (AVE), Insuficiência Cardíaca (IC) e Doença Renal Crônica (DRC) (BARROSO WKS, et al., 2021; MALTA DC, et al., 2017).

A prevalência da HAS no Brasil tem aumentado a cada ano. A doença afeta 32,5\%-de pessoas na fase adulta e mais de $60 \%$ na terceira idade, sendo responsável por um aumento de $50 \%$ das mortes por Doença Cardiovascular (DCV). Esse crescimento pode estar associado ao aumento da expectativa de vida da população brasileira, associado à exposição a fatores de risco modificáveis como: sedentarismo, hábitos alimentares irregulares, etilismo e uma rotina estressante (BARROSO WKS, et al., 2021; LOBO LAC, et al., 2017).

O tratamento da HAS consiste em duas medidas: farmacológica e não farmacológica. Na primeira, a terapia é feita com uso de medicamentos anti-hipertensivos e na segunda busca-se melhorar os hábitos de vida no que se refere ao padrão alimentar, principalmente no que diz respeito à diminuição do uso de sal, diminuição da ingestão de bebidas alcoólicas, aumento da prática regular de atividade física, diminuição do peso, em casos de obesidade e sobrepeso, redução da circunferência abdominal e da exposição a ambientes que possam gerar estresse (FALCÃO AS, et al., 2018).

O enfermeiro inserido na APS atua como o principal educador em saúde no controle da hipertensão arterial, de modo que atua informando o paciente sobre mudanças no estilo de vida, alimentação e tratamento correto, controlando a doença e prevenindo futuros eventos cardiovasculares (DIAS EG, et al., 2016).

Com o foco nas medidas não farmacológicas, a educação em saúde realizada pelos profissionais de saúde, dentre eles o Enfermeiro, voltada para promoção em saúde e prevenção de agravos, deve ser considerada uma estratégia importante para alcançar a conscientização da população. Nesse contexto, o enfermeiro é o protagonista na realização das atividades educativas, sendo responsável por articular e elaborar as práticas de acordo com a necessidade do público-alvo que pretende alcançar (BARRETO ACO, et al., 2019).

A educação em saúde é definida como o processo educativo que ocorre entre o profissional e o usuário, conforme necessidades desse indivíduo, buscando construir conhecimentos sobre determinado assunto acerca da saúde e dar a essa paciente autonomia sobre seu estado de saúde. Logo, essas ações vão dar espaço a reflexões e estímulos de mudança de hábitos e práticas, promovendo assim a saúde integral aos usuários e prevenindo agravos de seu estado de saúde-doença (SANTILLI PGJ, et al., 2016). Dessa forma, a atividade educativa em saúde tem grande relevância na mudança de estilo de vida. Por meio dessa estratégia, o enfermeiro busca informar e sensibilizar sobre a HAS (GONÇALVES RS, et al., 2020).

As ações voltadas para os hipertensos orientam-se para o empoderamento do indivíduo no seu autocuidado, melhoria da qualidade de vida e controle da hipertensão e suas complicações (BARRETO ACO, et al., 2019). O estímulo ao pensamento crítico possibilita aos hipertensos uma reflexão sobre a importância da adesão ao tratamento farmacológico e não farmacológico para controle e prevenção dos agravos (GONÇALVES RS, et al., 2020).

A Atenção Primária à Saúde (APS), considerada a porta de entrada no Sistema Único de Saúde (SUS), tem um papel fundamental no controle dessa patologia e na prevenção de agravos. As ações educativas desenvolvidas nesse cenário têm grande impacto no controle da pressão arterial e na redução dos casos de internação por doenças do aparelho cardiovascular como consequência da pressão alta (GIRÃO ALA, et al., 2016).

Em busca de medidas efetivas e modificadoras de hábitos de vida que visam melhor e maior tempo de vida para os hipertensos, é indispensável que o enfermeiro conheça a realidade e a cultura da população adscrita para elaborar e planejar atividades educativas que considerem o contexto de vida do indivíduo e da sua família (MAIA JD, et al., 2018). 
A partir do exposto, o presente estudo tem como objetivo conhecer as estratégias empregadas pelo enfermeiro da atenção primária na educação em saúde de hipertensos.

\section{MÉTODOS}

Trata-se de uma revisão integrativa, fundamentada na síntese e análise dos resultados de pesquisas científicas publicadas sobre as estratégias educativas utilizadas pelo enfermeiro na atenção primária para o enfrentamento da hipertensão arterial. As etapas que envolvem a sua construção são as seguintes: formulação de uma questão de pesquisa com relevância; seleção de estudos nas bases de dados a partir de critérios de inclusão e exclusão; análise e categorização dos estudos; interpretação dos resultados e apresentação dos resultados (MENDES KD, et al., 2008).

A revisão integrativa na área da enfermagem possibilita uma prática baseada em evidências à partir de um compilado de pesquisas sobre temas relevantes para a prática clínica, impactando diretamente na qualidade do cuidado prestado (SOUSA LMM, et al., 2017).

Para elaboração da questão de pesquisa, utilizou-se a estratégia PICO por possibilitar a elaboração de uma pergunta bem construída para a definição correta das informações necessárias à resolução da questão clínica pesquisada, sendo P de "população estudada" (enfermeiros da atenção primária); o I de "intervenção" (não se aplica); o C de "comparação" (não se aplica, pois não é um estudo comparativo) e $O$ de "desfecho" (estratégias educativas) (DONATO H e DONATO M, 2020). A questão de revisão foi: quais são as estratégias educativas utilizadas pelo enfermeiro da atenção primária voltadas para o enfrentamento da hipertensão arterial?

Foram investigadas as publicações científicas indexadas nas respectivas bases de dados: Cumulative Index to Nursing and Allied Health Literature (CINAHL), Medical Literature Analysis and Retrieval System Online (MEDLINE/PubMed), Literatura Latino-Americana e do Caribe em Ciências de Saúde (LILACS) e Scientific Electronic Library Online (SCIELO). Estas foram acessadas via login institucional da Universidade Federal de Juiz de Fora para obter conteúdo assinado do Portal de Periódicos da Coordenação de Aperfeiçoamento de Pessoal de Nível Superior (CAPES) a fim de alcançar um número maior de produções com texto completo.

A coleta foi realizada nos meses de setembro e outubro de 2020, com uso de palavras-chave selecionadas nos Descritores em Ciências da Saúde (DECs): 1\# Educação em saúde/Health Education; 2\# Enfermagem/Nursing; 3\# Hipertensão/Hipertension; 4\# Atenção Primária à Saúde/Primary Health Care. Os cruzamentos utilizados na busca foram: (1\# AND 2\# AND 3) e (1\# AND 2\# AND 3\# AND 4\#).

Foram incluídas as evidências publicadas na literatura nacional, incluindo teses, dissertações e artigos de periódicos online com o texto disponível para acesso gratuito, cujo foco tenha sido as estratégias educativas utilizadas pelo enfermeiro na atenção primária para o enfrentamento da hipertensão arterial; produções que retratavam a realidade do Brasil; adultos e idosos, compreendendo a faixa etária de 19 a mais de 80 anos; publicações nos idiomas português, espanhol e inglês. Utilizou-se recorte temporal de 20 anos a fim de alcançar as evidências mais recentes. Foram excluídos: resenhas, editoriais e revisão da literatura. A Tabela 1 revela o quantitativo de publicações identificadas, após aplicação dos filtros.

Tabela 1 - Sistematização da busca eletrônica nos portais de busca: CINAHL, MEDLINE/PubMed, LILACS E SCIELO Brasil.

\begin{tabular}{|c|c|c|c|c|}
\hline Descritores & LILACS & SCIELO & MEDLINE & CINAHL \\
\hline $\begin{array}{l}\text { Educação em saúde and Enfermagem and } \\
\text { Hipertensão }\end{array}$ & 114 & 47 & - & 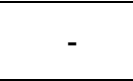 \\
\hline Health education and Nursing and Hipertension & - & - & 724 & 37 \\
\hline $\begin{array}{l}\text { Educação em saúde and enfermagem and } \\
\text { Hipertensão and Atenção Primária à Saúde }\end{array}$ & - & 6 & - & - \\
\hline $\begin{array}{l}\text { Health education and Nursing and Hipertension and } \\
\text { Primary Health Care }\end{array}$ & - & - & 58 & 1 \\
\hline Subtotal & 114 & 53 & 782 & 38 \\
\hline Total: & & & & \\
\hline
\end{tabular}

Fonte: Santos FAC, et al., 2021. 
Foram identificados, inicialmente, 987 registros por meio da busca nos portais LILACS, SCIELO, CINAHL e MEDLINE/PUBMED. Após a leitura do título e do resumo foram excluídas as produções científicas que não estavam em consonância com o tema proposto, assim como as duplicações, elegendo 28 estudos para leitura na íntegra. Após uma análise minuciosa do material, oito artigos foram incluídos na revisão. O fluxograma apresentado na Figura 1 revela o processo de identificação, seleção e inclusão dos estudos, segundo recomendações PRISMA (GALVÃO TF, 2015).

Figura 1 - Fluxograma do processo de identificação, seleção e inclusão dos estudos.
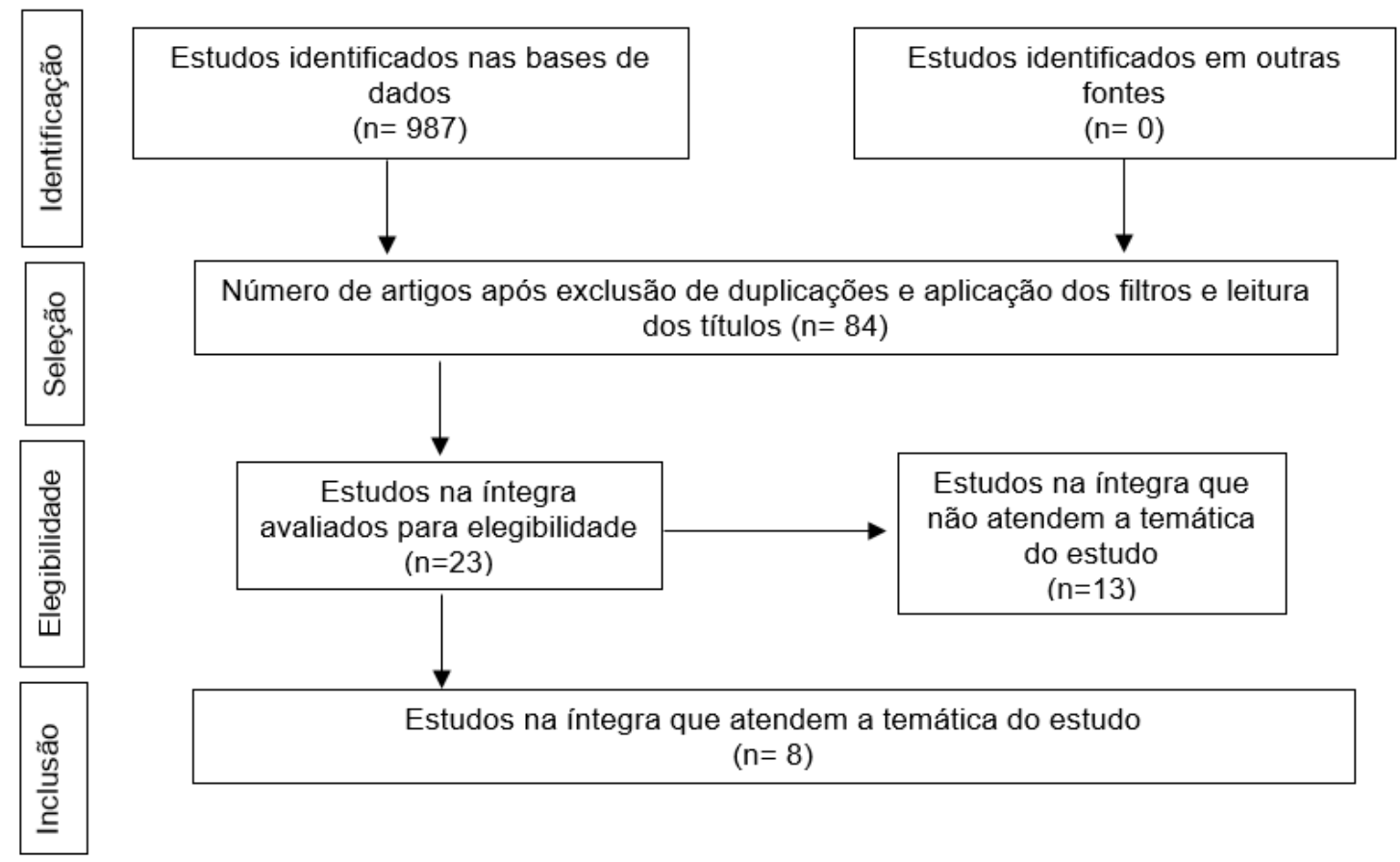

Número de artigos após exclusão de duplicações e aplicação dos filtros e leitura dos títulos $(n=84)$

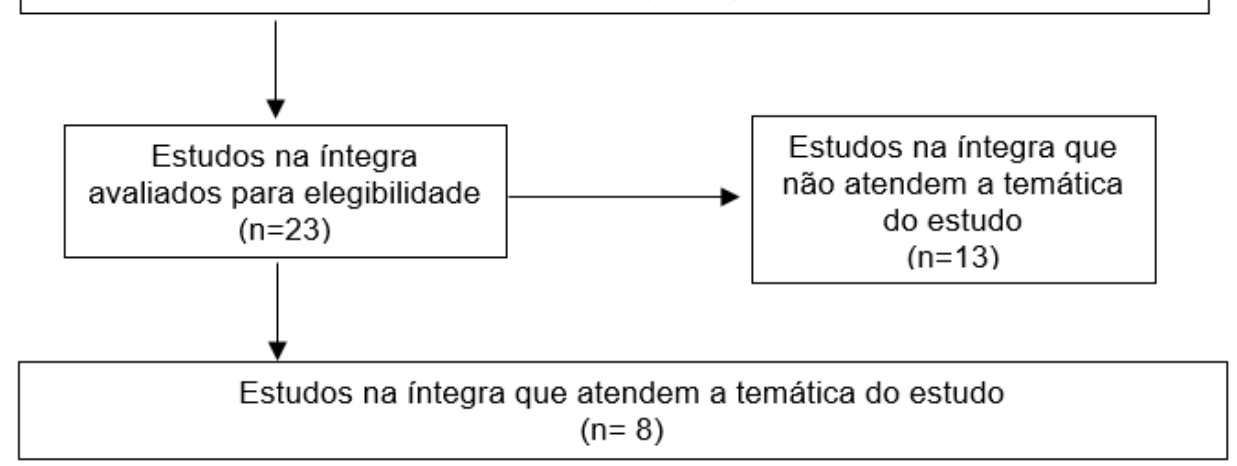

Fonte: Santos FAC, et al., 2021.

Em relação à definição do nível de evidência do estudo, utilizou-se a classificação da Agency for Health care Research and Quality (AHRQ) (2016), a qual estabelece: nível I, meta-análise de ensaios clínicos controlados; nível II, estudos experimentais individuais; nível III, ensaios quase experimentais; nível IV, estudos descritivos ou qualitativos; nível V, relato de caso ou experiência; nível VI, opinião de expertises.

Para a caracterização dos dados, assim como o desenvolvimento da análise e interpretação das publicações que compuseram a amostra, foi utilizado um formulário com os seguintes tópicos: título; autores; ano da publicação; periódico; população e cenário de estudo; desenho do estudo e método; objeto de estudo; resultado; implicações; nível de evidência.

\section{RESULTADOS}

O presente estudo compreendeu oito estudos nacionais. Quanto ao ano de publicação, percebeu-se um longo período sem publicações sobre o tema, sendo a última produção no ano de 2015. Com relação ao nível de evidência dos estudos, identificou-se um de nível III, seis de nível IV e um de nível V. Os estudos primários que compuseram essa revisão foram identificados por um código alfanumérico, sendo constituído pela letra A, seguido de um número que corresponde a ordem decrescente do ano de publicação (de A1 a A8).

Depreendeu-se dos resultados que o enfermeiro utiliza estratégias grupais, sala de espera, visita domiciliar e a consulta do Hiperdia para educação em saúde da pessoa com hipertensão arterial. Os estudos revelam que, muitas vezes, essa educação ainda é pautada na transmissão vertical e ressalta sobre a importância do estabelecimento do vínculo e do diálogo para que a sensibilização da pessoa com HAS seja efetiva. O Quadro 1 destaca as publicações que constituem essa revisão com os principais resultados encontrados e 0 respectivo nível de evidência (NE). 
Quadro 1: Estudos primários selecionados para revisão

\begin{tabular}{|c|c|c|c|c|}
\hline Código & Autores (ano) & Título & Resultados & Nível de Evidência \\
\hline $\mathrm{A} 1$ & $\begin{array}{l}\text { Bezerra GC, et al. } \\
(2015) .\end{array}$ & $\begin{array}{l}\text { Implementation of hiperdia group in a family } \\
\text { health unit: an experience report. }\end{array}$ & $\begin{array}{l}\text { As estratégias educativas desenvolvidas para promoção da saúde ocorrem em } \\
\text { palestras. }\end{array}$ & $\mathrm{V}$ \\
\hline $\mathrm{A} 2$ & $\begin{array}{l}\text { Silva FM, et al. } \\
\qquad(2014) \text {. }\end{array}$ & $\begin{array}{l}\text { Contribuições de grupos de educação em } \\
\text { saúde para o saber de pessoas com } \\
\text { hipertensão. }\end{array}$ & $\begin{array}{l}\text { A educação em saúde é desenvolvida por meio de atividades em grupo, com } \\
\text { discussões temáticas referentes às temáticas de saúde. A metodologia } \\
\text { expositiva, se dava por meio de palestras, foi vista pelos participantes como } \\
\text { estratégia pedagógica que tem sucesso na fundamentação do ensino sobre } \\
\text { saúde. }\end{array}$ & IV \\
\hline A3 & $\begin{array}{l}\text { Carvalho FPB, et al. } \\
\text { (2013). }\end{array}$ & $\begin{array}{l}\text { Popular education in health with the group } \\
\text { hiperdia of a basic health unit. }\end{array}$ & $\begin{array}{l}\text { A educação em saúde desenvolvida na consulta do Hiperdia, na maior parte dos } \\
\text { casos, não envolve o pensamento crítico, devido à grande quantidade de usuários } \\
\text { que o enfermeiro precisa atender. É importante ressaltar que a transmissão } \\
\text { vertical ainda consiste na prática dos enfermeiros, baseado em um modelo de } \\
\text { prescrição, onde o enfermeiro orienta e o usuário segue. No entanto, a educação } \\
\text { pautada no diálogo na saúde é a melhor solução para atender as necessidades } \\
\text { do paciente. }\end{array}$ & IV \\
\hline A4 & $\begin{array}{l}\text { Silva FVF, et al. } \\
\text { (2013). }\end{array}$ & $\begin{array}{l}\text { Cuidado de enfermagem a pessoas com } \\
\text { hipertensão fundamentado na teoria de } \\
\text { Parse. }\end{array}$ & $\begin{array}{l}\text { A estratégia utilizada na educação em saúde é uma prática focada na escuta das } \\
\text { dificuldades dos usuários e na resolução desses problemas por meio do cuidado } \\
\text { integral. Se não ocorrer um vínculo inicial efetivo, é comum ocorrer o abandono e } \\
\text { problemas na adesão ao tratamento. }\end{array}$ & IV \\
\hline A6 & $\begin{array}{l}\text { Silva RV, et al. } \\
\qquad(2008)\end{array}$ & $\begin{array}{l}\text { Vivência de educação em saúde: o grupo } \\
\text { enquanto proposta de atuação. }\end{array}$ & $\begin{array}{l}\text { Trabalhar a educação em saúde em grupos permite a integração entre os } \\
\text { membros e o enfermeiro, esse momento de modo a respeitar os seus saberes e, } \\
\text { principalmente, desenvolver a escuta que condiciona o sucesso dessa forma de } \\
\text { trabalho. }\end{array}$ & IV \\
\hline A7 & $\begin{array}{l}\text { Mantovani MF, et al. } \\
\text { (2007). }\end{array}$ & $\begin{array}{l}\text { Visita domiciliar de enfermagem com } \\
\text { atividades educativas no tratamento da } \\
\text { pressão arterial. }\end{array}$ & $\begin{array}{l}\text { Valer-se das visitas domiciliares como estratégia de educação em saúde contribui } \\
\text { para que os usuários se percebam importantes para a equipe de saúde, além de } \\
\text { permitir o esclarecimento de dúvidas surgem durante o tratamento. }\end{array}$ & III \\
\hline A8 & $\begin{array}{l}\text { Rolim MO e Castro } \\
\text { ME (2007). }\end{array}$ & $\begin{array}{l}\text { Adesão ao programa de controle da } \\
\text { hipertensão e aos resultados padronizados } \\
\text { de enfermagem: um estudo exploratório. }\end{array}$ & $\begin{array}{l}\text { A estratégia educativa é a interação entre o paciente e o enfermeiro. As } \\
\text { informações devem ser compartilhadas de forma individual, respeitando as } \\
\text { necessidades e atendendo as expectativas do paciente, sem imposições. O } \\
\text { enfermeiro deve respeitar as "visões de mundo" dos usuários no que diz respeito } \\
\text { ao seu convívio social e cultural. }\end{array}$ & IV \\
\hline
\end{tabular}

Fonte: Santos FAC, et al., 2021. 


\section{DISCUSSÃO}

A educação em saúde é um processo de construção de conhecimento em que o profissional aborda temáticas de saúde com o intuito de promover a saúde e prevenir doenças e seus agravos a um indivíduo ou uma população (RAMOS CFV, et al., 2018).

Nesse contexto, o enfermeiro da atenção básica deve analisar as questões sociais do meio em que realiza as práticas educativas para que esse processo aconteça de forma dinâmica e que alcance os objetivos desejados, como o desenvolvimento da autonomia do paciente no seu processo de cuidar. Para o desenvolvimento dessas atividades, o profissional irá atuar de forma pedagógica e com planejamento para garantir a manutenção da saúde de uma determinada comunidade de forma efetiva (RAMOS CFV, et al., 2018).

A atividade educativa em saúde tem grande importância na adesão e no sucesso do tratamento da hipertensão arterial sistêmica, visto que o enfermeiro vai conhecer aquele indivíduo e vai direcioná-lo ao tratamento adequado, monitorando seu estado de saúde e evitando possíveis agravos (SILVA FHM, et al., 2019). No contexto das práticas educativas realizadas com os hipertensos, segundo o estudo $A 5$, o enfermeiro da atenção primária enquanto educador em saúde, exerce um papel ativo na elaboração de estratégias que mobilizam as pessoas para o controle da doença (JÚNIOR JEM, et al., 2011).

Na educação em saúde com foco no controle da HAS o enfermeiro tem a oportunidade de propor mudanças nos hábitos de vida do indivíduo por meio da conscientização sobre seu processo de saúdedoença. Para isso, ao elaborar as atividades educativas, deve considerar o meio em que a pessoa vive e o seu saber popular, dessa forma o sujeito se sentirá mais à vontade para compartilhar e dividir com o profissional o que melhor se ajusta ao seu cotidiano, segundo sua cultura, costumes e condições socioeconômicas (ARNEMANN CT, et al., 2018).

Os estudos A1, A2, A3, A4 e A5 corroboram com os autores supracitados ao afirmarem que uma estratégia a ser utilizada pelo enfermeiro da atenção primária durante as atividades educativas, visando eficácia no processo de adesão às mudanças de hábitos de vida e tratamento adequado, é considerar as questões culturais e sociais das pessoas envolvidas (BEZERRA GC, et al., 2014; SILVA FM, et al., 2014; CARVALHO FPB, et al., 2013; SILVA FVF, et al., 2013; JÚNIOR JEM, et al., 2011).

Segundo o estudo A3, a educação dialógica é uma estratégia que facilita o processo de ensinoaprendizagem sobre as mudanças de hábitos que são necessárias após o diagnóstico da doença. $A$ conscientização não é passiva, mas envolve a pessoa no seu autocuidado (CARVALHO FPB, et al., 2013). O estudo A8 reforça a importância de o diálogo ser pautado no respeito, calma e compreensão, para que a atividade educativa aconteça com êxito e possa gerar resultados positivos (ROLIM MO e CASTRO ME, 2017).

Levando em consideração os saberes da pessoa, o diálogo aberto entre o enfermeiro e os hipertensos durante as atividades educativas possibilitará o estabelecimento do vínculo, o que reverbera em uma maior confiança e, consequentemente, uma melhor adesão ao tratamento (MAGRI S, 2020).

Para isso, segundo o estudo A1 é preciso romper paradigmas instituídos em nossa sociedade como o profissional de saúde detentor de todo o conhecimento e promover a educação em saúde de forma ativa, de modo que a pessoa se torne protagonista em seu autocuidado (BEZERRA GC, et al., 2014). Nesse cenário, o enfermeiro deve utilizar de técnicas que tornam essas atividades mais singulares e pessoais considerando as questões culturais que envolvem cada indivíduo. A construção compartilhada do conhecimento vai favorecer o comprometimento da pessoa no controle da HAS e seus possíveis agravos (BARRETO ACO, et al., 2019).

Para o sucesso da educação em saúde é importante evitar termos e técnicas de linguagem biomédica, visto que, precisa-se incluir a pessoa como sujeito ativo no seu tratamento, com capacidade de cuidar de si e alcançar o controle da HAS (SOARES AN, et al., 2017). Segundo o estudo A5, empoderar a pessoa adoecida a coloca como protagonista do seu tratamento, uma estratégia importante para adesão às orientações em saúde que visam uma melhor qualidade de vida (JÚNIOR JEM, et al., 2011). 
Os estudos A2 e A3 ressaltam a importância de informar o indivíduo sobre suas condições clínicas, pois compreender o processo de adoecimento pela HAS vai favorecer um melhor controle dos fatores que causam o agravamento dessa condição (SILVA FM, et al., 2014; CARVALHO FPB, et al., 2013). Dessa maneira, o enfermeiro vai interagir com o paciente de forma direta para informá-lo sobre seu estado de saúde e para realizar as devidas orientações para que ocorra adesão ao tratamento. Por isso é tão importante a relação de proximidade entre o enfermeiro e o hipertenso, para o alcance das metas terapêuticas. Os grupos educativos desenvolvidos na atenção primária são importantes estratégias para alcançar o controle da doença por meio da conscientização (SALLES ALO, et al., 2019).

Os estudos A1 e A2 enfatizam as estratégias grupais com atividades lúdicas e ativas para discussões aprofundadas sobre as questões relacionadas à hipertensão e seus possíveis agravos, destacando-se a palestra como um meio de comunicação efetivo (BEZERRA GC, et al., 2014; SILVA FM, et al., 2014). Segundo Souza E, et al. (2018), por meio da palestra é possível incentivar mudanças nos padrões alimentares, estimular a atividade física e melhorar a adesão ao uso de medicamentos anti-hipertensivos, pautando-se na troca de saberes entre o hipertenso e o enfermeiro.

A sala de espera também é evidenciada nos estudos $A 1, A 2$ e A6 como estratégia educativa para informar os hipertensos e pessoas saudáveis sobre a hipertensão, tratamento da doença e das complicações. Acreditase que essa abordagem deve ser sustentada de forma lúdica por meio de teatros e cartazes, além de debates, grupos de conversa e até mesmo simulação realística. (BEZERRA GC, et al., 2014; SILVA FM, et al, 2014; SILVA RV, et al., 2008).

A sala de espera tem um grande potencial de conscientização das pessoas que buscam a unidade básica de saúde, uma vez que enquanto aguardam a consulta médica, o enfermeiro utiliza cartazes, exposição de objetos e imagens, e até mesmo vídeos para abordar sobre a hipertensão e seus possíveis agravos, enfatizando a importância da prevenção e tratamento precoce. Dessa forma, a sala de espera pode propiciar um ambiente agradável e educativo para aqueles que aguardam as consultas (NEGRÃO MLB, et al., 2018).

Além das atividades grupais, a consulta individual do Hiperdia também se destaca como uma estratégia para efetivar ações educativas. $\mathrm{O}$ estudo $\mathrm{A} 3$ aponta que apesar de não ser suficiente devido à alta demanda de usuários para um só enfermeiro, a consulta constitui um meio de encontro em que a educação em saúde se torna possível, momento oportuno para dialogar sobre as possíveis complicações da HAS e os fatores de risco que o indivíduo está exposto (CARVALHO FPB, et al., 2013).

Dessa forma, a consulta de enfermagem propicia o cuidado individualizado, favorecendo a mudança de comportamento e a adesão aos tratamentos farmacológicos e não farmacológicos. Quando esse encontro, permeado pelo diálogo e troca de saberes, consegue promover a conscientização dos hipertensos, os resultados são satisfatórios em relação a melhora do quadro clínico, visto que essa é uma prática segura e garantida de promoção à saúde (MOTA BAM, et al., 2019).

Em uma abordagem individual, o enfermeiro tem possibilidades de elaborar um plano de cuidados individualizado e atividades educativas sistematizadas e moldadas segundo a realidade da pessoa, já que isso é fundamental para adesão ao tratamento (PINTO ESO e RODRIGUES WN, 2018). Nesse sentido, o estudo A4 reitera que a educação em saúde deve ser planejada conforme as necessidades do sujeito (SILVA FVF, et al., 2013).

Ao se tratar de uma abordagem individualizada, pode-se destacar a Visita Domiciliar (VD) ao hipertenso como momento oportuno do enfermeiro explorar a realidade daquele indivíduo e fornecer as orientações necessárias para melhor êxito no tratamento da hipertensão, haja vista o seu contexto de vida (A7) (MANTOVANI MF, et al., 2007).

Durante a VD o enfermeiro vai se aproximar da realidade do hipertenso, observando o seu ambiente, seus hábitos e possíveis mudanças nesse cenário. Ao se inserir no âmbito residencial do sujeito, é possível elaborar um plano de cuidados e realizar a educação em saúde mais condizente com as condições socioeconômicas daquela família, por isso a VD é considerada um instrumento tão importante pelas equipes de saúde (NASCIMENTO JS, 2013). 
Identifica-se como limitação da presente revisão poucos estudos relacionados ao tema, o que dificultou a obtenção de resultados, mesmo utilizando um recorte temporal de 20 anos. Soma-se ao fato de as produções selecionadas refletirem somente a realidade brasileira da Atenção Primária à Saúde.

\section{CONSIDERAÇÕES FINAIS}

As diferentes estratégias educativas utilizadas pelo enfermeiro na atenção básica visam empoderar o hipertenso no seu autocuidado e têm sido utilizadas para o enfrentamento da HAS, como: palestras, sala de espera, visita domiciliar e consulta de Hiperdia. Espera-se, a partir desse estudo, incentivar a discussão ainda no ensino da graduação sobre as possibilidades de estratégias educativas que empoderem a população no seu autocuidado. Nota-se que a presente revisão apresentou um número baixo de artigo que versam sobre 0 tema, acredita-se que a escolha de produções científicas somente nacionais, a fim de retratar a realidade brasileira, tenha contribuído para esse quantitativo.

\section{REFERÊNCIAS}

1. AGENCY FOR HEALTH CARE RESEARCH AND QUALITY (AHRQ). Quality Improvement and monitoring at your fingertips. Rockville: Agency for Healthcare Research and Quality; 2016.

2. ARNEMANN CT, et al. Educação em saúde e educação permanente: ações que integram o processo educativo da enfermagem. Rev. baiana enferm. 2018; 32: 1-10.

3. BARRETO ACO, et al. Perception of the Primary Care multiprofissional team on health education. Rev Bras Enferm. 2019; 72(sppl1): 266-273.

4. BARROSO WKS, et al. Diretrizes Brasileiras de Hipertensão Arterial - 2020. Arq. Bras. Cardiol. 2021; 114(3): 516658.

5. BEZERRA GC, et al. Implementation of hiperdia group in a family health unit: an experience report. Rev. Enferm. Digit. Cuid. Promoção da Saúde. 2014; (1): 19-22.

6. CARVALHO FPB, et al. Educação popular em saúde com o grupo hiperdia de uma unidade básica de saúde. Rev. enferm. UFPE on line. 2013; 7(8): 5157-164.

7. DIAS EG, et al. Contribuições da enfermagem na adesão ao tratamento da hipertensão arterial: uma revisão integrativa da literatura brasileira. Rev de Epidem e Cont de Infec. 2016; 6(3): 138-144.

8. DONATO H, DONATO M. Etapas na condução de uma revisão sistemática. Acta Med Port [internet]. 2020; 32(3): 227235.

9. FALCÃO AS, et al. Estilo de vida e adesão ao tratamento de hipertensão arterial sistêmica em homens idosos. Rev. bras. promoç. saúde. 2018; 31(2): 1-10.

10. GALVÃO T, et al. Principais itens para relatar revisões sistemáticas e meta-análises: a recomendação PRISMA. Epidemiol. Serv. Saúde. 2015; 24(2): 335-42.

11. GIRÃO ALA, et al. Usuários hipertensos na atenção primária à saúde: acesso, vínculo e acolhimento à demanda espontânea. Rev Gaúcha Enferm. 2016; 37(2): e60015.

12. GONÇALVES RS, et al. Health education as a strategy for the prevention and promotion of health in a basic health unit. Braz. J. Hea. Rev. 2020; 3(3): 5811-17.

13. JUNIOR JEM, et al. Educação em saúde como estratégia para melhoria da qualidade de vida dos usuários hipertensos. Rev Rene (Online). 2011; 12: 1045-51.

14. LOBO LAC, et al. Tendência temporal da prevalência de hipertensão arterial sistêmica no Brasil. Cad Saúde Pública. 2017; 33(6).

15. SALLES ALO, et al. O enfermeiro e a questão da adesão do paciente ao tratamento da hipertensão arterial sistêmica. Rev Enferm UERJ. 2019; 27(0): 37193.

16. MAGRI S, et al. Programa de educação em saúde melhora indicadores de autocuidado em diabetes e hipertensão. RECIIS (Online). 2020; 14(2): 386-400.

17. MAIA JD, et al. Educação em saúde para usuários hipertensos: percepções de profissionais da estratégia saúde da família. Rev. Ciênc. Plur. 2018; 4(1): 81-97.

18. MALTA DC, et al. Prevalence of and factors associated with self-reported high blood pressure in Brazilian adults. Rev Saúde Públ. 2017; 51: 1-11.

19. MANTOVANI MF, et al. Visita domiciliar de enfermagem com atividades educativas no tratamento da pressão artérial. Online Brazilian J Nurs. 2007; 6(1).

20. MENDES KD, et al. Revisão integrativa: método de pesquisa para a incorporação de evidências na saúde e na enfermagem. Texto \& contexto enferm. 2008; 4(17): 758-764. 
21. MOTA BAM, et al. Efetividade da consulta de enfermagem na adesão ao tratamento da hipertensão arterial sistèmica. Rev. salud pública [Internet]. 2019; 21(3): e370291.

22. NASCIMENTO JS, et al. Visitas domiciliares como estratégias de promoção da saúde pela enfermagem. Rev. bras. promoç. saúde. 2013; 26(4): 513-22.

23. NEGRÃO MLB, et al. The waiting room: potential for people with arterial hypertension to learn. Rev Bras Enferm. 2018; 71(6): 3105-12.

24. PINTO ESO, RODRIGUES WN. Sistematização da Assistência de Enfermagem na Atenção Primária à pessoa portadora de hipertensão arterial. Nursing (São Paulo). 2018; 21: 2036-40.

25. RAMOS CFV, et al. Education practices: research-action with nurses of Family Health Strategy. Rev Bras Enferm. 2018; 71(3): 1144-51.

26. ROLIM MO, CASTRO ME. Adesão às orientações fornecidas no programa de controle da hipertensão: uma aproximação aos resultados padronizados de enfermagem. Online Brazilian J Nurs. 2007; 6(1): 15-27.

27. SANTILLI PGJ, et al. Educação em saúde: algumas reflexões sobre sua implementação pelas equipes da estratégia saúde da família. Revista brasileira de promoção da saúde. 2016; 29: 1806-1230.

28. SILVA FM, et al. Contributions of health education groups to the knowledge of people with hypertension. Rev Bras Enferm. 2014; 67(3): 347-53.

29. SILVA FVF, et al. Cuidado de enfermagem a pessoas com hipertensão fundamentado na teoria de Parse. Esc. Anna Nery Rev. Enferm. 2013; 17(1): 111-9.

30. SILVA FHM, et al. Intervenção de saúde sobre hipertensão e diabetes. Rev. enferm. UFPE on line. 2019;13: e240593.

31. SILVA RV, et al. Vivência de educação em saúde: o grupo enquanto proposta de atuação. Trab educ e saúde (Online). 2008; 6(3): 633-44

32. SOARES AN, et al. Dispositivo educação em saúde: reflexões sobre práticas educativas na atenção primária e formação em enfermagem. Texto \& contexto enferm. 2017; 26(3):1-9.

33. SOUSA LMM, et al. A metodologia de revisão integrativa da literatura em enfermagem. Revista investigação em enfermagem. 2017; 21(2): 17-26.

34. SOUZA E, et al. Educação em saúde a portadores de hipertensão e diabetes na atenção primária. Nursing. 2018; 21(240): 2178-83. 